\title{
Analyst
}

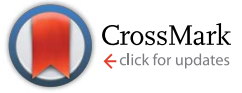

Cite this: Analyst, 2014, 139, 5614

DOI: $10.1039 / c 4 a n 90075 h$

www.rsc.org/analyst

\section{Correction: Exploring the origins of the apparent "electrocatalytic" oxidation of kojic acid at graphene modified electrodes $\dagger$}

\author{
Luiz C. S. Figueiredo-Filho, ${ }^{b}$ Dale A. C. Brownson, ${ }^{a}$ Orlando Fatibello-Filho ${ }^{b}$ \\ and Craig E. Banks*a
}

Correction for 'Exploring the origins of the apparent "electrocatalytic" oxidation of kojic acid at graphene modified electrodes' by Luiz C. S. Figueiredo-Filho et al., Analyst, 2013, 138, 4436-4442.

The text that appears on page 4437 under the heading 'Experimental section' that reads:

ESI Fig. S1A $\dagger$ depicts a typical Transmission Electron Microscope (TEM) image of the commercially purchased graphene and ESI Fig. S1B $\dagger$ shows a high resolution TEM image where a hexagonal arrangement of carbon atoms, which is characteristic of graphene, is clearly evident.

should be changed to:

ESI Fig. S1A $\dagger$ depicts a typical TEM image of a graphene sheet that has been fabricated using the same method as our commercially sourced graphene and Fig. S1B $\dagger$ shows a high-resolution TEM image (from the same source) where a hexagonal arrangement of carbon atoms, which is characteristic of graphene, is clearly evident. Independent TEM and Raman analysis of the commercially sourced graphene (as received from the supplier and consequently as used throughout this work) is presented in Fig. S3 and S4† respectively.

The Royal Society of Chemistry apologises for these errors and any consequent inconvenience to authors and readers. 